\title{
Identification of the potential oncogenes in glioblastoma based on bioinformatic analysis and elucidation of the underlying mechanisms
}

\author{
YONG ZHANG, QIMING XIA and JUN LIN \\ Department of Neurosurgery, The People's Hospital of Guizhou Provincial, Guiyang, Guizhou 550002, P.R. China
}

Received December 18, 2017; Accepted May 31, 2018

DOI: $10.3892 /$ or.2018.6483

\begin{abstract}
Glioblastoma (GBM) is a common malignant tumour in the human brain, but its molecular mechanisms have not been systematically evaluated. The aim of this study was to identify potential key oncogenes associated with the progression of GBM and to elucidate their mechanisms. The gene expression profile of GSE50161, selected from the Gene Expression Omnibus database, was analysed to find cancer-associated genes and gene functions in GBM. In total, 486 differentially expressed genes, including 128 upregulated genes, were identified. The function and pathway enrichment of these genes were analysed through Gene Ontology and Kyoto Encyclopedia of Genes and Genomes analyses. Survival analysis for three selected partially upregulated genes, CDK1, CCNB1 and CDC20, showed that their high expression was significantly associated with poor survival in GBM. CDK1 was selected for validation of its function and molecular mechanism in GBM. This gene was significantly overexpressed in GBM cancer tissues and cells compared with normal control cells. In addition, knockdown of CDK1 clearly inhibited GBM cell proliferation. Notably, we demonstrated that CDK1 was involved in the Akt signalling pathway, where it promotes the process involved in GBM malignancy.
\end{abstract}

\section{Introduction}

Glioblastoma (GBM) is an aggressive cancer of glial cells and accounts for $80 \%$ of all adult primary malignant brain tumours (1). Despite advances in surgical techniques, radiotherapy and chemotherapy for its treatment, the prognosis of GBM patients remains poor dut to its complex pathogenesis $(2,3)$. There is substantial evidence that many

Correspondence to: Professor Jun Lin, Department of Neurosurgery, The People's Hospital of Guizhou Provincial, 83 Zhongshang East Road, Nanming, Guiyang, Guizhou 550002, P.R. China

E-mail: fgehu149@126.com

Key words: glioblastoma, oncogenes, CDK1, proliferation, Akt oncogenes or tumour-suppressor genes are involved in the progression of GBM and that the effects of these genes often depend on the level of their transcription, which can be regulated by gene mutations, epigenetic modifications or non-coding RNAs (4-6). However, the dysregulated gene networks and pathways that lead to GBM progression remain unclear, and the molecular mechanisms underlying GBM have not yet been elucidated. Thus, the identification of new oncogenes and establishing the underlying molecular mechanisms for GBM are critically important and highly needed.

Microarray technology has now become an important tool for revealing global gene expression changes to identify the genes involved in carcinogenesis, including GBM. The widespread application of gene chips has produced large amounts of microarray data, which have been deposited and stored in public databases such as the Gene Expression Omnibus (GEO) database (https://www.ncbi.nlm.nih.gov/geo/) hosted by the US National Center for Biotechnology Information. This database serves as a public genetic expression profile repository for a wide range of microarray data. In the present study, we downloaded the mRNA expression profile of GSE50161 from the GEO database and used bioinformatics methods to compare GBM and normal brain samples to identify the differentially expressed genes (DEGs). Gene ontology (GO) and pathway enrichment analyses were applied to these DEGs using the DAVID bioinformatics resource (https://david.ncifcrf.gov/).

Cyclin-dependent kinases (CDKs), including three interphase CDKs (CDK2, CDK4 and CDK6) and a mitotic CDK (CDK1), are important regulators of cell cycle progression and cell cycle regulation (7). Deregulation of this family of proteins is a hallmark of several diseases, including cancer, and emerging evidence has demonstrated that specific interphase CDKs are required for tumour cell proliferation (8). CDK1 is activated by type A cyclin at the metaphase to promote mitogenesis, and it has been shown that, among the cell cycle CDKs, CDK1 is sufficient for driving the cell cycle of all cell types, indicating its master role in cell proliferation regulation (8). It has been reported that $\mathrm{CDK} 1$ is overexpressed in several human cancers and that it is associated with poor survival or the malignancy processes of those cancers (9-11). However, the role and mechanism of CDK1 in GBM and its development have not been fully elucidated. 


\section{Materials and methods}

Microarray data. We extracted the gene expression profile of GSE50161 from the GEO database. GSE50161 was submitted by Griesinger et al (12) and was based on the platform of GPL570 (Affymetrix Human Genome U133 Plus 2.0 Array; Affymetrix Inc., Santa Clara, CA,USA). The GSE50161 dataset contains 130 samples, including 34 GBM and 13 normal brain tissues.

Data preprocessing and differentially expressed gene analysis. The probe-level data in CEL files were converted into expression measures; then, using the default parameters in the R Affy package (http://www.bioconductor.org/packages/release/bioc/html/affy.html), a robust multiple array averaging (RMA) algorithm was applied to background correct and quartile normalise the data (13). The significance of DEGs were evaluated with $\mathrm{R}$ statistical software (version 3.4.1; https://www.r-project.org/) and Bioconductor analysis tools (http://www.bioconductor.org/). The limma package in $\mathrm{R}$ was used to identify the genes that were differentially expressed between GBM and normal samples (14). To circumvent the multi-test problem, resulting in too many false-positive results, the raw P-values of the genes were adjusted with the Benjamin and Hochberg method. The genes with adjusted $\mathrm{P}<0.05$ and $\mid \log \mathrm{FCl}>2$ were considered to be differentially expressed.

GO and pathway enrichment analysis of the DEGs. GO analysis is commonly used in functional studies of large-scale transcriptomic or genomic data (15), with the Kyoto Encyclopedia of Genes and Genomes (KEGG) knowledge database used to identify functional and metabolic pathways. In this study, we divided the DEGs into upregulated and downregulated groups and then used the Database for Annotation, Visualization and Integrated Discovery (DAVID) to perform functional enrichment analysis for the identified DEGs with a threshold of FDR $<0.05$. Pathway analysis based on the KEGG database was performed with a threshold value of adjusted $\mathrm{P}<0.05$.

Cell culture. The malignant glioma cell lines U-87MG, U-251MG and SHG44 were purchased from the Chinese Academy of Sciences (Shanghai, China) and STR profiles were presented for all cell lines used. The malignant glioma cell line U-138MG and the human normal brain glial cells HEB were sourced from the American Type Culture Collection (ATCC, Manassas, VA, USA). The cells were maintained in high-glucose DMEM medium (Hyclone Laboratories, Logan, UT, USA) containing $10 \%$ fetal bovine serum (FBS; Gibco, Grand Island, NY, USA) and were cultured at $37^{\circ} \mathrm{C}$ in humidified air with $5 \% \mathrm{CO}_{2}$.

RNA extraction and quantitative real-time polymerase chain reaction ( $R T-q P C R)$. Total RNA was extracted from GBM cells using TRIzol reagent (Takara Biotechnology, Co., Ltd., Dalian, China), following the manufacturer's protocol. cDNA was synthesised using PrimeScript ${ }^{\mathrm{TM}}$ RT Master Mix (Takara Biotechnology), according to the manufacturer's instructions. The sequences of primers were as follows: CDK1 forward,
5'-CAGTCAGACCAAAATACCTACTGGGT-3' and reverse, 5'-ACACCAACCAGCTGCAGCATCTTCTT-3'. RT-qPCR assays were performed with SYBR Green Master Mix kit (Takara Biotechnology) using a Real-Time PCR System (Applied Biosystems, Foster City, CA, USA). Reaction steps were as follows: $95^{\circ} \mathrm{C}$ for $30 \mathrm{sec}, 40$ cycles with $95^{\circ} \mathrm{C}$ for $5 \mathrm{sec}$, $60^{\circ} \mathrm{C}$ for $30 \mathrm{sec}$, and $95^{\circ} \mathrm{C}$ for $10 \mathrm{sec}, 65^{\circ} \mathrm{C}$ for $5 \mathrm{sec}$, end of $95^{\circ} \mathrm{C}$ to $10.5^{\circ} \mathrm{C}$. GAPDH was used as internal control and the $2^{-\Delta \mathrm{Cq}}$ (or $2^{-\Delta \Delta \mathrm{Ct}}$ ) method was used to analyse the relative quantitation of gene expression levels.

Protein extraction and western blot analysis. Total protein was extracted from GBM cells using RIPA (Beyotime Institute of Biotechnology, Shanghai, China). Protein samples were separated by electrophoresis on SDS-PAGE gel of $10 \%$ and transferred onto polyvinylidene fluoride (PVDF) membranes. The membranes were blocked in 5\% skim milk at room temperature for $2 \mathrm{~h}$, and then incubated at $4^{\circ} \mathrm{C}$ overnight with primary antibodies CDK1 (1:2,000 dilution; cat. no. ab18; Abcam, Cambridge, UK), p-AKT (1:1,000 dilution; cat. no. 4060; Cell Signalling Technology, Danvers, MA, USA), cyclin A (1:1,000 dilution; cat. no. 4656; Cell Signalling Technology), cyclin B (1:1,000 dilution; cat. no. 4138; Cell Signalling Technology) and $\beta$-actin (1:3,000 dilution; cat. no. 3700; Cell Signalling Technology). The membranes were washed with TBST buffer and incubated in secondary antibodies with horseradish peroxidase (1:3,000 dilution; cat. no. 14709; Cell Signalling Technology) at room temperature for $2 \mathrm{~h}$. Finally, the expression of proteins was visualised with an ECL reagent (Bio-Rad Laboratories, Shanghai, China). $\beta$-actin was used as the internal control, and the experiments were performed in triplicate. Quantitation of proteins expression was analysed by Quantity One 4.6.2 software (Bio-Rad Laboratories, Foster City, CA, USA).

Transfection and stably transfected cells. CDK1-specific small hairpin RNA (shRNA) sequence and the overexpression sequence of CDK1 were constructed using a lentiviral technique from Shanghai GeneChem Co. (Shanghai, China). The CDK1 shRNA sequences were as follows: shRNA1, 5'-CAC CGGTTCCTAGTACTGCAATTCGCGAACGAATTGCAGT ACTAGGAACC-3'; shRNA2, 5'-CACCGGATGTGCTTA TGCAGGATTCCGAAGAATCCTGCATAAGCACATCC-3'; shRNA3, 5'-CACCGCAGGATTCCAGGTTATATCTCG AAAGATATA ACCTGGAATCCTGC-3'. U-87MG and U-251MG cells were transfected with lentiviral diluent and selected with $3 \mu \mathrm{g} / \mathrm{ml}$ puromycin (Sigma-Aldrich; Merck KGaA, Darmstadt, Germany) for one week. The knockdown efficiency of CDK1 was identified by RT-qPCR and western blot assays.

Cell proliferation assays. Cell Counting Kit-8 (CCK-8; Dojindo Laboratories, Kumamoto, Japan) was used to detected cell proliferation. The cells were seeded into 96-well plates, with $0.5 \times 10^{4}$ cells/well. CCK- 8 reagent (at a final concentration up to $10 \%$ ) was added to each well at $0,24,48$ and $72 \mathrm{~h}$ after complete cell adherence. The reaction system was incubated at $37^{\circ} \mathrm{C}$ for $1.5 \mathrm{~h}$, and the absorbance was measured at $450 \mathrm{~nm}$ with a microplate reader (BioTek Instruments, Inc., Winnoski, VT, USA). The experiments were conducted in triplicate. 
Table I. Top 20 upregulated DEGs in GBM compared with normal brain tissues.

\begin{tabular}{|c|c|c|c|}
\hline Gene symbol & Gene name & $\operatorname{logFC}$ & Adjusted P-value \\
\hline RRM2 & Ribonucleotide reductase regulatory subunit M2 & 4.453419652 & $5.51 \mathrm{E}-18$ \\
\hline PBK & PDZ binding kinase & 4.83243913 & $1.00 \mathrm{E}-17$ \\
\hline CKLF-CMTM1 & CKLF-CMTM1 readthrough & 2.037217181 & 8.61E-17 \\
\hline TSPAN6 & Tetraspanin 6 & 2.238197957 & $2.42 \mathrm{E}-16$ \\
\hline TOP2A & Topoisomerase (DNA) II $\alpha$ & 3.649973973 & $2.69 \mathrm{E}-16$ \\
\hline GPX7 & Glutathione peroxidase 7 & 2.299383845 & $3.04 \mathrm{E}-16$ \\
\hline PRDX4 & Peroxiredoxin 4 & 2.006805339 & $3.34 \mathrm{E}-16$ \\
\hline TYMS & Thymidylate synthetase & 2.217762746 & $2.29 \mathrm{E}-15$ \\
\hline KIF11 & Kinesin family member 11 & 3.25308997 & $2.41 \mathrm{E}-15$ \\
\hline KIAA0101 & KIAA0101 & 2.434042313 & $2.42 \mathrm{E}-15$ \\
\hline ACTL6A & Actin like 6A & 2.334230872 & $2.71 \mathrm{E}-15$ \\
\hline NDC80 & Kinetochore complex component & 3.801758823 & $3.73 \mathrm{E}-15$ \\
\hline BUB1B & BUB1 mitotic checkpoint serine/threonine kinase B & 3.326136589 & $4.61 \mathrm{E}-15$ \\
\hline KIF4A & Kinesin family member $4 \mathrm{~A}$ & 3.186827599 & $5.43 \mathrm{E}-15$ \\
\hline CDK1 & Cyclin dependent kinase 1 & 2.632518398 & $8.08 \mathrm{E}-15$ \\
\hline UHRF1 & Ubiquitin like with PHD and ring finger domains 1 & 3.829808433 & $8.68 \mathrm{E}-15$ \\
\hline WEE1 & WEE1 G2 checkpoint kinase & 2.605771805 & $1.09 \mathrm{E}-14$ \\
\hline CDCA7L & Cell division cycle associated 7 like & 2.502772251 & $1.46 \mathrm{E}-14$ \\
\hline $\mathrm{EZH} 2$ & Enhancer of zeste 2 polycomb repressive complex 2 subunit & 3.33804613 & 3.34E-14 \\
\hline RAD51AP1 & RAD51 associated protein 1 & 2.586126911 & $3.59 \mathrm{E}-14$ \\
\hline
\end{tabular}

DEGs, differentially expressed genes; GBM, glioblastoma.

Colony formation assays. In brief, cells were seeded into 6 -well plates, at $0.1 \times 10^{4}$ cells per well, and maintained for two weeks in a medium containing $10 \%$ FBS. When the clones exceeded 50 cells, the cells were fixed with $4 \%$ paraformaldehyde and stained with $1 \%$ crystal violet. Finally, the colonies were imaged by scanner (Samsung, Gyeonggi-do, Korea) and counted by the counting tool of Photoshop 6.0 (Adobe Photoshop, San Jose, CA, USA). The experiment was conducted in triplicate.

Statistical analysis. The overall survival analysis used Kaplan-Meier curves based on data from the GEO and The Cancer Genome Atlas (TCGA) databases. Hazard ratios (HRs) with 95\% confidence intervals were calculated, and the log-rank test was used to assess P-values, and these are displayed on the plot. Data are presented as the mean \pm standard deviation (SD) from triplicate independent experiments, and were analysed with Student's t-test for two group comparisons, and were analysed by analysis of variance (ANOVA) followed by Dunnett or Bonferroni post hoc test for multiple comparisons. A two-tailed P-value $<0.05$ was considered to indicate a statistically significant difference. The data analysis was performed using SPSS version 20.0 (IBM Corp., Armonk, NY, USA).

\section{Results}

Identification of DEGs. We downloaded the GSE50161 gene expression dataset from the GEO database. Following careful inspection, we extracted 45 microarrays based on the GPL570 platform, including 34 GBM samples and 13 normal brain samples. Based on the cut-off criteria of adjusted $\mathrm{P}<0.05$ and $\mid \log \mathrm{FCl}>2$, a total of 486 DEGs were identified, of which 128 were upregulated and 358 were downregulated. Fig. 1 displays the top 50 upregulated genes as a heat map, and Table I lists details of the top 20 upregulated DEGs.

Functional and pathway enrichment analysis of DEGs. DAVID was used to identify highly enriched GO categories and KEGG pathways from all the upregulated DEGs. The GO analysis identified 57 significant enrichments of upregulated DEGs, which were classified into three GO categories: Biological processes, cellular components, and molecular functions (Fig. 2A). In the biological process category, the upregulated DEGs were particularly enriched in cell division, mitotic nuclear division and cell proliferation. In the cellular components category, the upregulated DEGs were enriched in midbodies, nucleoplasm, cytoplasm and nuclei. The GO molecular functions analysis also showed that the upregulated DEGs were significantly enriched in protein binding, identical protein binding, ATP binding and protein kinase binding. In addition, the pathway enrichment analysis showed that a total of 8 KEGG pathways were enriched, with cell cycle the most highly enriched pathway (Fig. 2B).

Partial upregulated genes were significantly associated with poor survival. The upregulated DEGs involved in the cell cycle signalling pathway were CDK1, CCNB1, TTK, BUB1B, CDC20, MCM2, CDK4, CCNA2 and WEE1. Among these, 


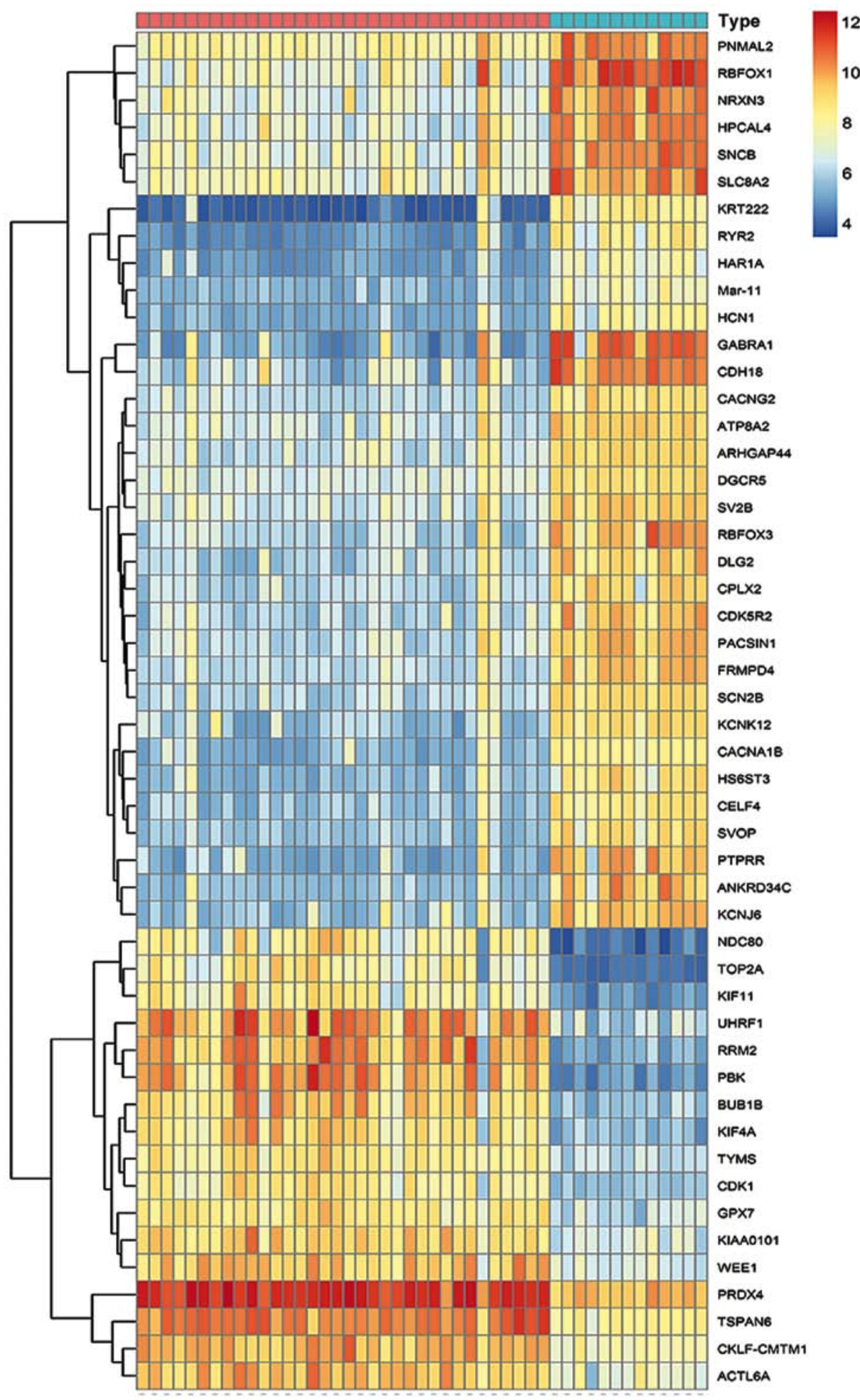

Figure 1. Heat map of the top 50 DEGs that were significantly upregulated or downregulated in samples from patients with GBM compared to normal brain samples. DEGs, differentially expressed genes; GBM, glioblastoma.

CDK1, CCNB1 and CDC20 were also enriched in the most significant function of GO. Therefore, we selected these three DEGs and evaluated the relationship between their expression and the survival prognosis of patients with GBM. Gene expression data and clinical information from the GEO and TCGA databases were used to investigate the prognostic significance of these genes. The result showed that high expression of CDK1 (Fig. 3A and B), CDC20 (Fig. 3C and D), and CCNB1
(Fig. 3E and F) was associated with a significantly poorer overall survival.

CDK1 is overexpressed in GBM tissues and cell lines. CDK1 mRNA expression in GBM and normal brain tissues was evaluated from GEO and TCGA databases, showing that CDK1 was significantly overexpressed in GBM tissues, as compared to that noted in the normal brain tissues 

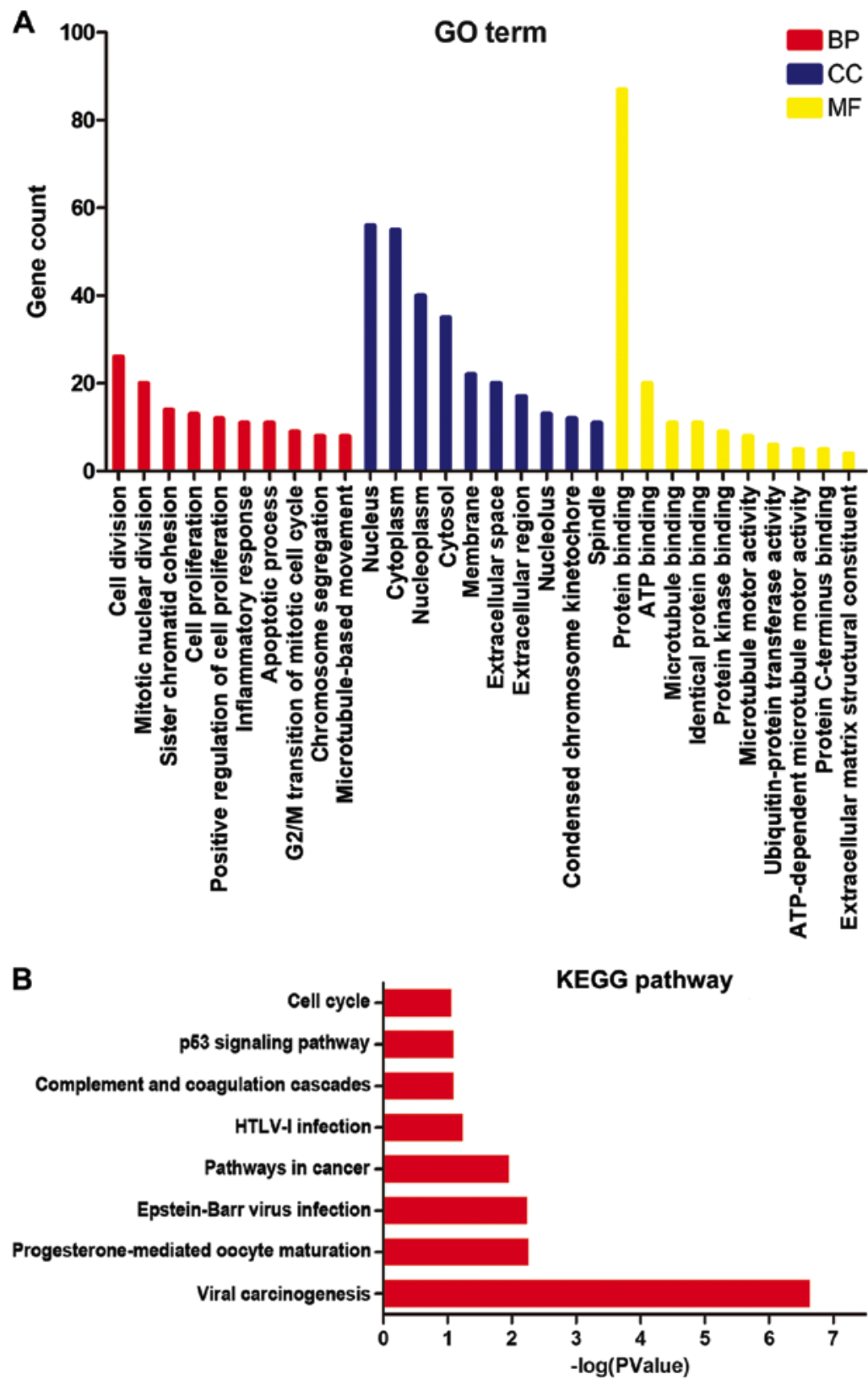

Figure 2. The gene ontology (GO) function and Kyoto Encyclopedia of Genes and Genomes (KEGG) pathway enrichment analysis. (A) GO analysis and the top 10 significant enriched GO terms of upregulated differentially expressed genes (DEGs) in the molecular function, biological process and cellular component categories. (B) Significantly enriched KEGG pathway terms of the upregulated DEGs.

(Fig. 4A and B). Its expression was similarly examined in glioma cell lines. CDK1 expression was much higher in glioma cells than that in normal human brain glial cell line (HEB), especially in GBM cells U-87MG and U-251MG (Fig. 4C and D).

Knockdown of CDK1 significantly inhibits the proliferation of $G B M$ cells. To study the role of CDK1 in the progression of GBM, we knocked down CDK1 expression in U-87MG and U-251MG cells using a lentiviral shRNA technique. The knockdown efficiency was evaluated using RT-qPCR and western blot analysis. The results showed significantly lower CDK1 mRNA and protein expression in U-87MG cells transfected with shRNA1, shRNA2 and shRNA3 than in cells transfected with control shRNA (Fig. 5A and B). Similar results were found in U-251MG cells (Fig. 5C and D). The effect of CDK1 on the proliferation of GBM cells was assessed using CCK-8 cell proliferation and colony formation assays, which showed that knockdown of CDK1 significantly inhibited GBM cell proliferation (Fig. 5E and F). Similarly, the colony formation assays demonstrated a lower colony formation ability in the cells transfected with CDK1 shRNA than this ability noted in the control group (Fig. 5G and H). These findings indicated that CDK1 plays a vital role in the proliferation of GBM cells.

CDK1 is involved in the Akt signalling pathway, promoting the GBM malignancy process. It has been shown the dysregulation of the Akt signalling pathway is associated with tumorigenesis and the development of GBM (16). Previous study have reported that CDK1 could be a downstream molecule in the 

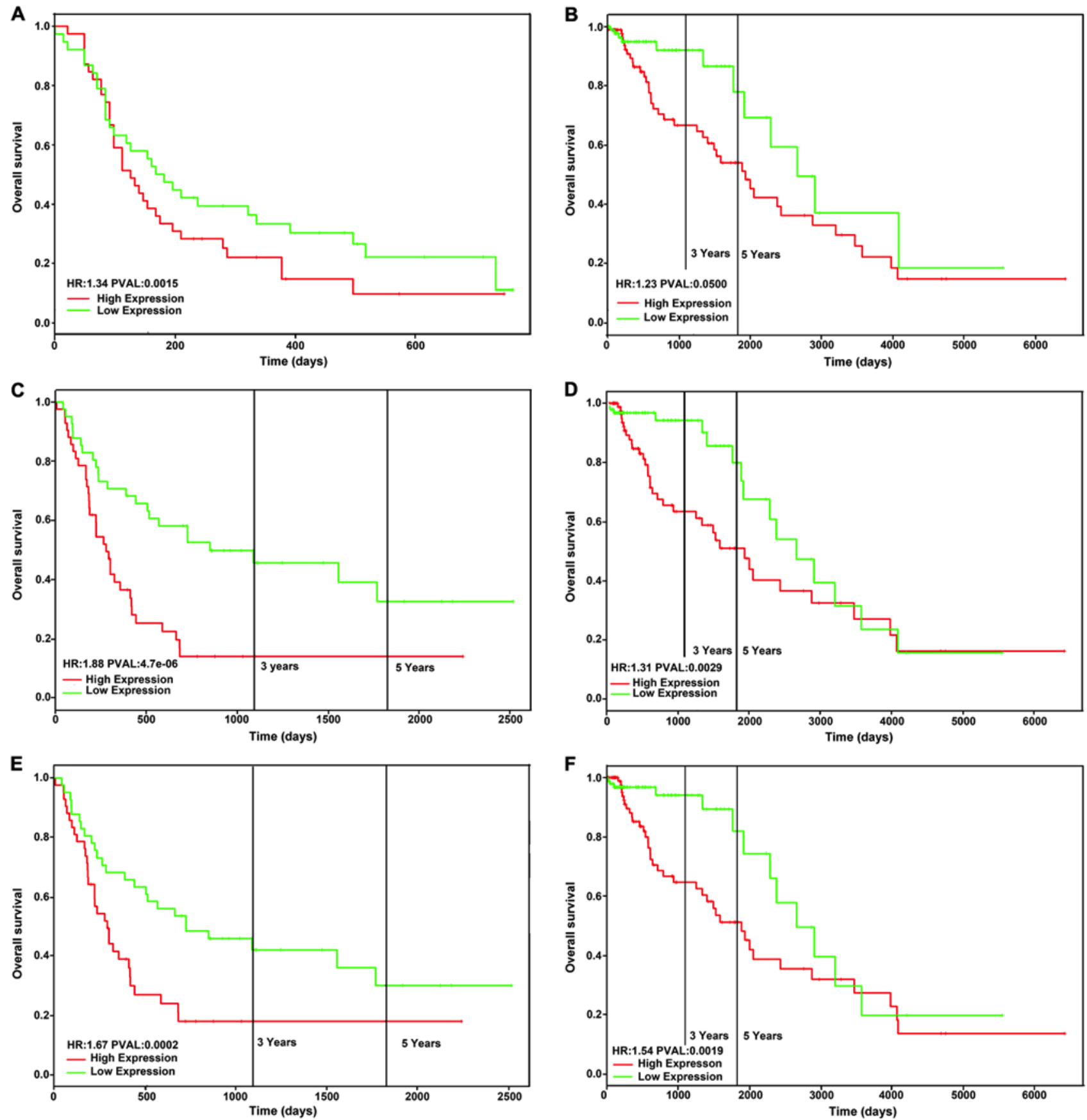

Figure 3. Kaplan-Meier analysis of the partial upregulated differentially expressed genes in patients with GBM. (A) Relationship between CDK1 expression and the survival of GBM patients analysed with the GEO databases. (B) Relationship between CDK1 expression and the survival of GBM patients analysed with The Cancer Genome Atlas (TCGA) databases. (C) Relationship between CDC20 expression and the survival of GBM patients analysed with GEO databases. (D) Relationship between CDC20 expression and the survival of GBM patients analysed with TCGA databases. (E) Relationship between CCNB1 expression and the survival of GBM patients analysed with GEO databases. (F) Relationship between CCNB1 expression and the survival of GBM patients analysed with TCGA databases. GBM, glioblastoma; GEO, Gene Expression Omnibus.

Akt signalling pathway (17). In the present study, we found that knockdown of CDK1 significantly inhibited the proliferation of GBM cells. To further demonstrate the involvement of CDK1 in the Akt signalling pathway-promoting GBM malignancy process, we treated U-87MG cells with IGF-1, an Akt signalling pathway agonist. p-Akt, proliferation-promoting factors, including cyclin $\mathrm{A}$ and cyclin $\mathrm{B}$, and CDK1 were significantly increased in the cells treated with IGF-1 when compared with levels noted in the untreated cells; this effect was observed in the negative control cells and in the CDK1
shRNA cells (Fig. 6A and B). We next inhibited the Akt signalling pathway in U-87MG cells by using a potent inhibitor of PI3K named GDC-0491. Western blot assays showed that p-Akt and CDK1 were significantly decreased in CDK1 overexpressed cells and negative control cells which were treated with GDC-0491, when compared with these levels in the untreated cells (Fig. 6C and D). These results indicate that CDK1 is a downstream molecule of the Akt signalling pathway. Finally, treatment with GDC-0491 obviously inhibited the proliferation of U-87MG cell, which could be reversed 

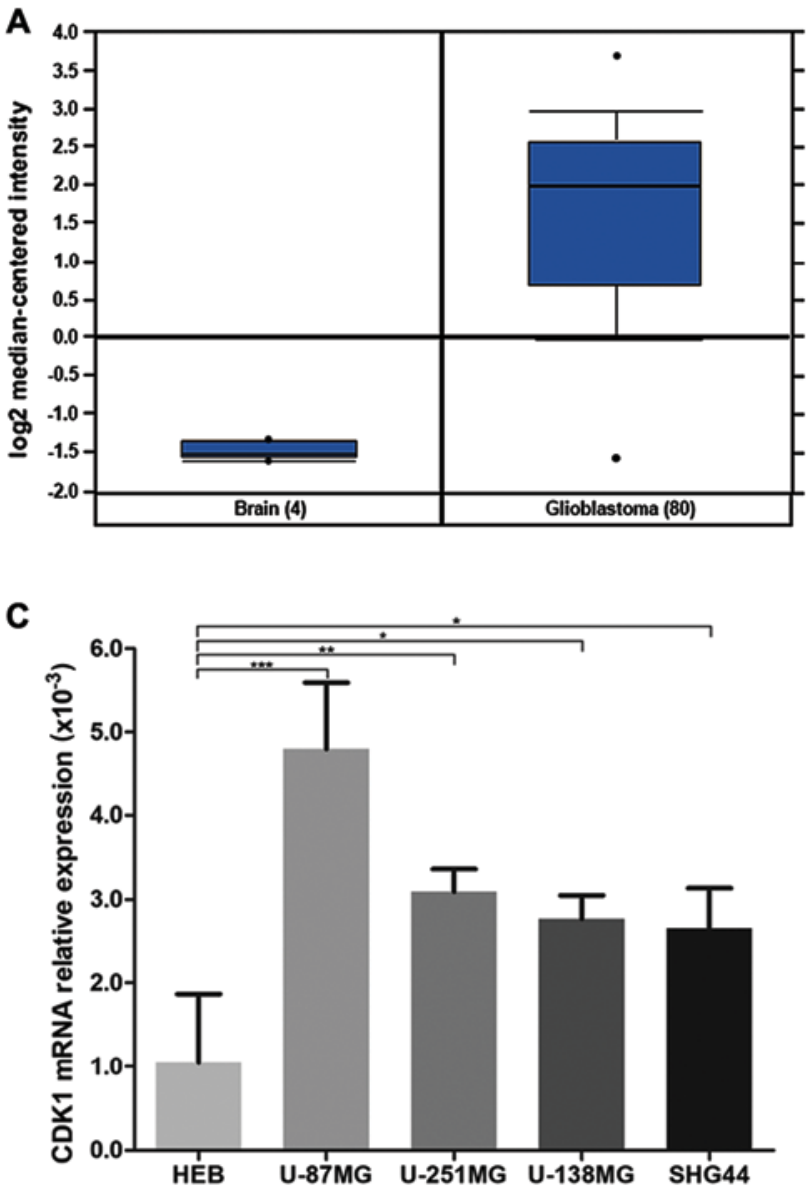

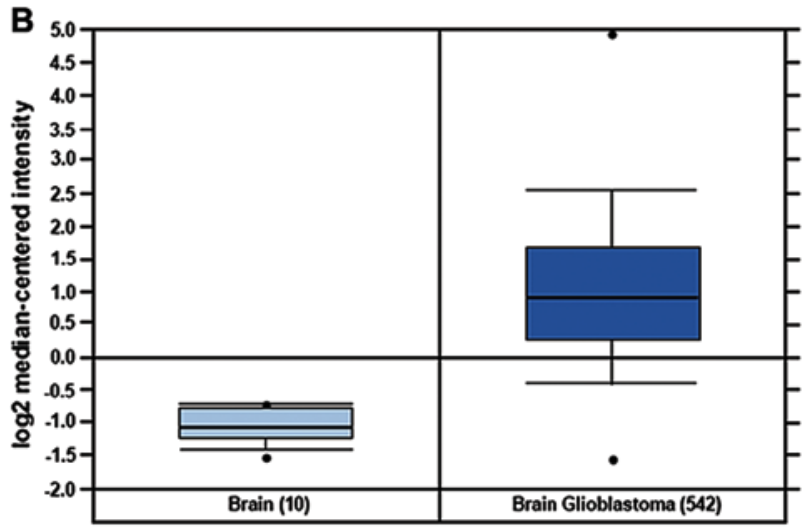

D

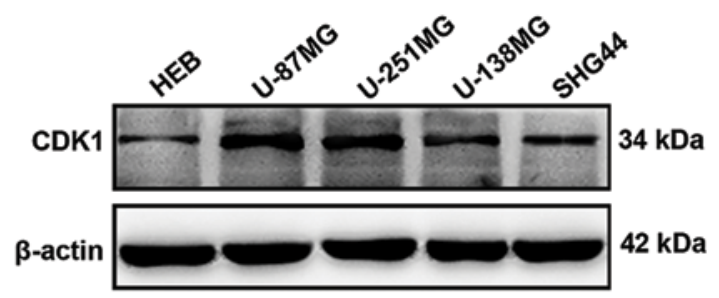

Figure 4. CDK1 was overexpressed in glioblastoma (GBM) tissues and cell lines. (A) CDK1 mRNA expression was analysed in the GEO databases. (B) CDK1 mRNA expression was collected from The Cancer Genome Atlas (TCGA) databases. (C) CDK1 mRNA expression was detected in glioma cells using qRT-PCR assays, ${ }^{*} \mathrm{P}<0.05,{ }^{* *} \mathrm{P}<0.01,{ }^{* * *} \mathrm{P}<0.0001$. (D) CDK1 protein expression was detected in glioma cells using western blot analysis. GBM, glioblastoma; GEO, Gene Expression Omnibus.

by the overexpression of CDK1 (Fig. 6E). In accordance with expectations, the CCK-8 assays showed that treatment with IGF-1 clearly promoted the proliferation of U-87MG cells, and that this was partially attenuated by the knockdown of CDK1 (Fig. 6F). In summary, this evidence demonstrated that CDK1 was involved in the Akt signalling pathway to promote GBM malignancy process.

\section{Discussion}

GBM is the most common malignant primary brain tumour in adults (18). Despite improvements in standard therapy, patients with GBM still have a poor prognosis, with a median survival of only about 15 months (19). Understanding the dysregulated gene networks and pathways leading to GBM is therefore necessary and urgent. In the present study, 486 DEGs were screened, including 128 upregulated genes and 358 downregulated genes. To obtain a fuller understanding of these DEGs, we performed GO function and KEGG pathway analyses. The GO analysis showed that the upregulated DEGs were primarily related to cell division, cell proliferation, midbodies, nucleoplasm, protein binding and protein kinase binding. The main KEGG pathways of the upregulated DEGs included the cell cycle, the p53 signalling pathway, complement and coagulation cascades and pathways in cancer. These analyses may provide new insights and a better understanding of the oncology of GBM.

CDK1, also known as cell division cycle protein 2 homolog, is a highly conserved protein that functions as a serine/threonine kinase. During cell proliferation, it plays a key role in $\mathrm{G}_{1} / \mathrm{S}$ and $\mathrm{G}_{2} / \mathrm{M}$ phase transitions, which promote the $\mathrm{M}$-phase process. It has been reported that CDK1 is involved in cancer tumourigenesis and development in various ways (20-22). In the present analysis, CDK1 was identified as an upregulated gene involved in cell division, cell proliferation and apoptotic processes, and was shown to be downstream of the cell cycle and p53 signalling pathways by GO and KEGG analysis. In addition, the high expression of CDK1 was significantly associated with poor overall survival in patients with GBM. Thus, CDK1 may play a vital role in the progression of GBM. Knockdown of CDK1 significantly inhibited GBM cell proliferation, confirming this hypothesis. This finding was also consistent with previous research into the role of CDK1 in other cancers, which showed that cancer cells with high CDK1 expression appeared to have greater cell proliferation capability (23-25).

Since its initial discovery as a proto-oncogene, Akt has attracted much attention due to its critical role in the regulation of diverse cellular functions, including metabolism, growth, proliferation, survival, transcription and protein synthesis (26). 

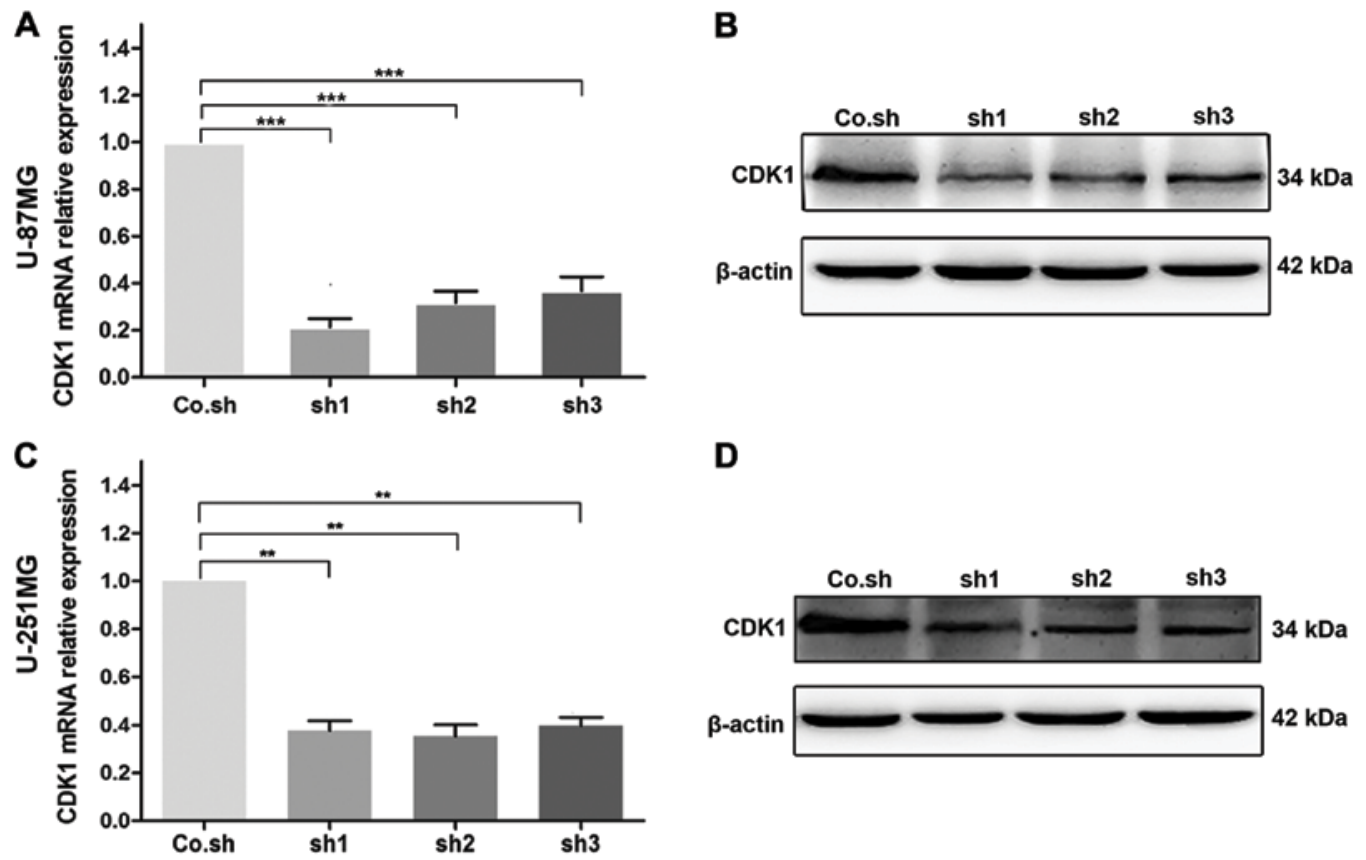

D

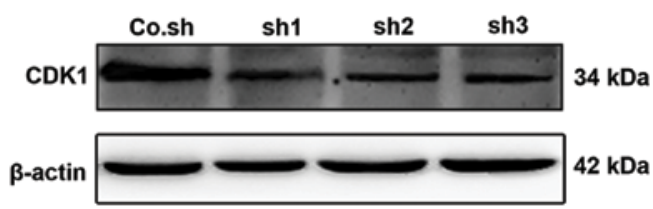

E

U-87MG

$\mathbf{F}$

U-251MG
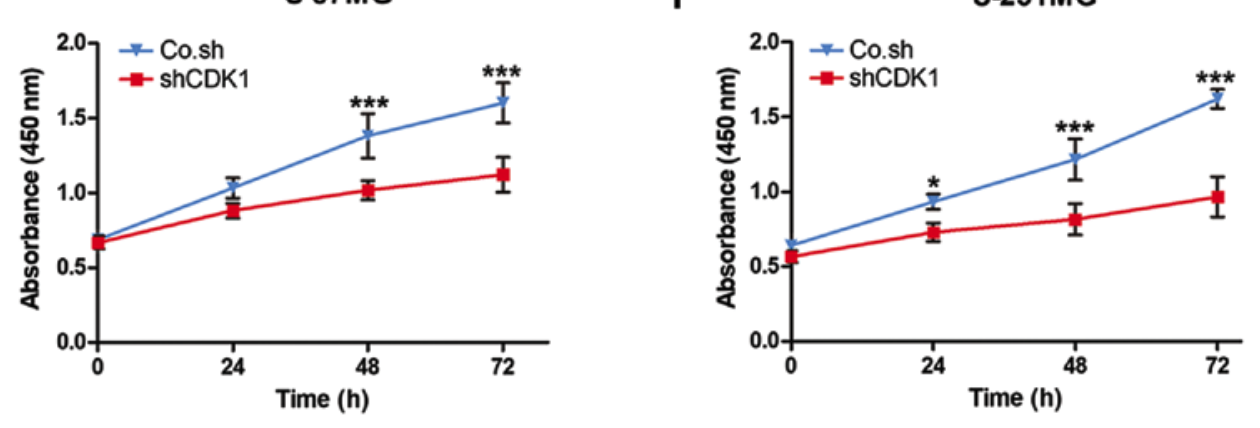

G

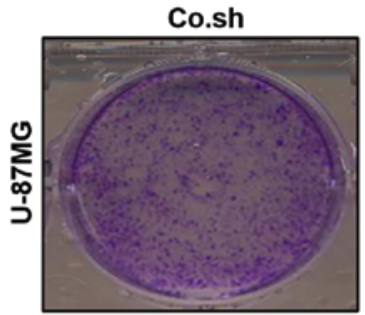

shCDK1


H
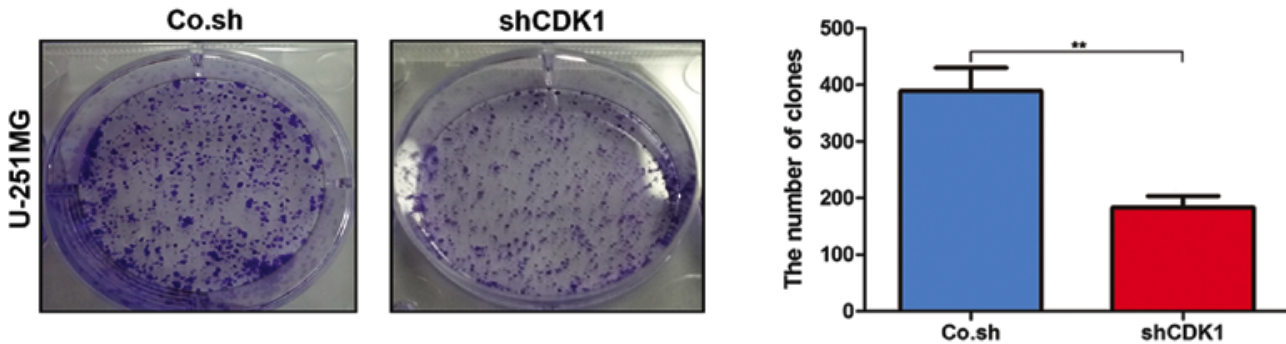

Figure 5. Knockdown of CDK1 significantly inhibited the proliferation of glioblastoma (GBM) cells. (A and B) The knockdown efficiency of CDK1 in U-87MG cell was examined by qRT-PCR and western blotting, ${ }^{* * *} \mathrm{P}<0.0001$. (C and D) The knockdown efficiency of CDK1 in U-251MG cell was examined by qRT-PCR and western blotting, ${ }^{* *} \mathrm{P}<0.01$. (E and F) The effect of CDK1 on GBM cell proliferation was detected by CCK-8 assays, ${ }^{*} \mathrm{P}<0.05,{ }^{* * * *} \mathrm{P}<0.0001$. $(\mathrm{G}$ and $\mathrm{H})$ The effect of $\mathrm{CDK} 1$ on GBM cell clone formation was detected by a clone formation assay, ${ }^{* *} \mathrm{P}<0.01$.

Deregulation of the Akt signalling pathway contributes to the development and progression of multiple solid tumours, including GBM $(27,28)$. In GBM, the pathway is regulated by multiple genetic events, mutations, amplifications, and deletions (29), and the activation of Akt seems to be a consequence of changes to its upstream molecules, such as PTEN, EGFR and PDK1 (16). However, its downstream in GBM is complex and has not yet been completely elucidated. In the present study, we activated 
A

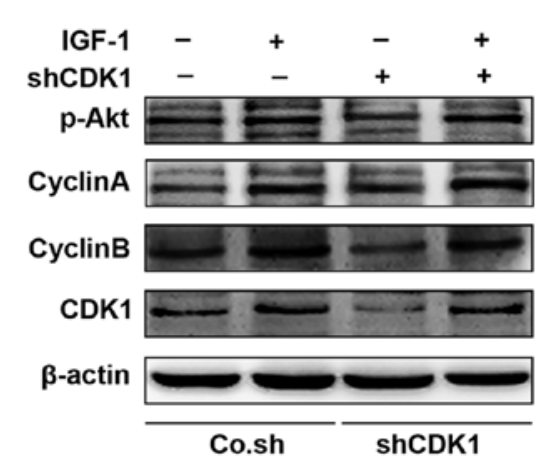

C

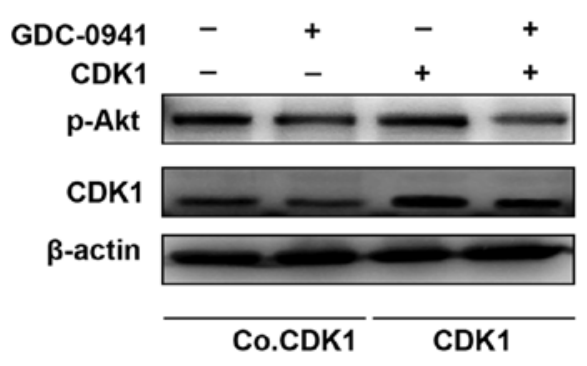

E

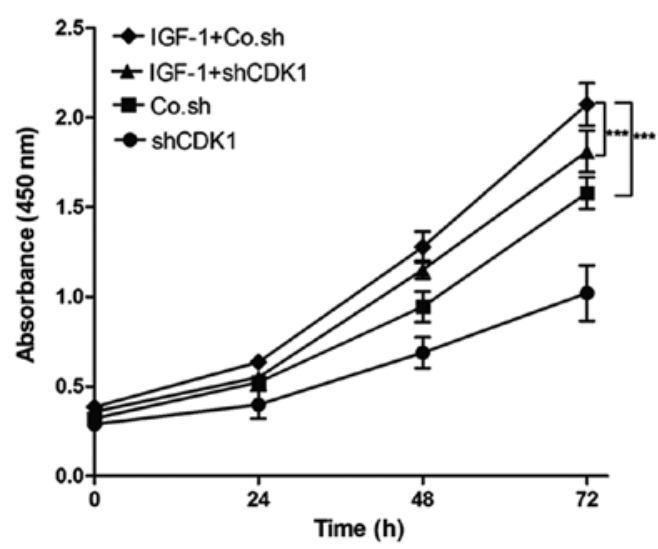

B

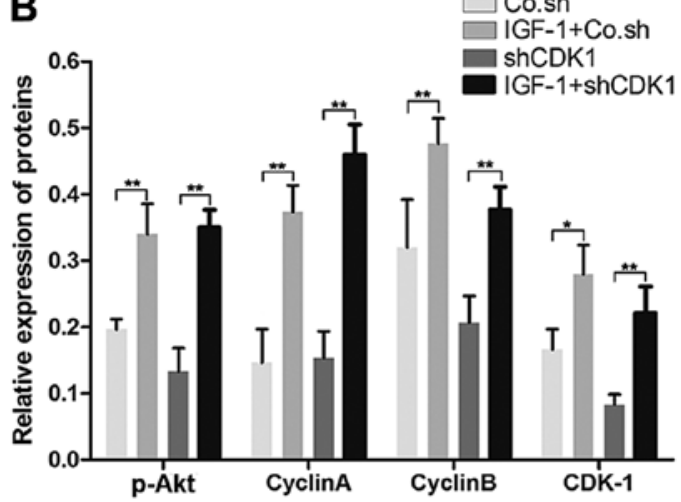

D

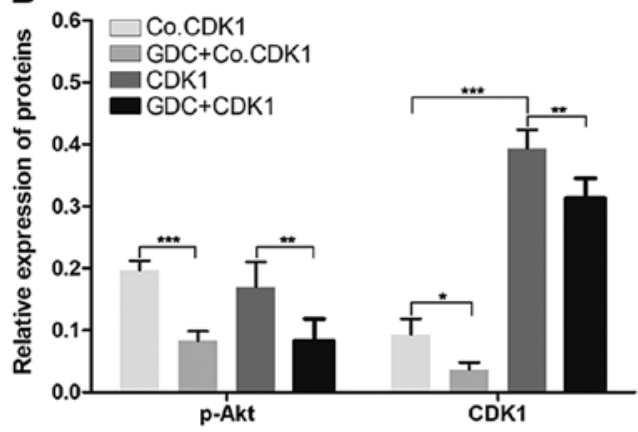

$\mathbf{F}$

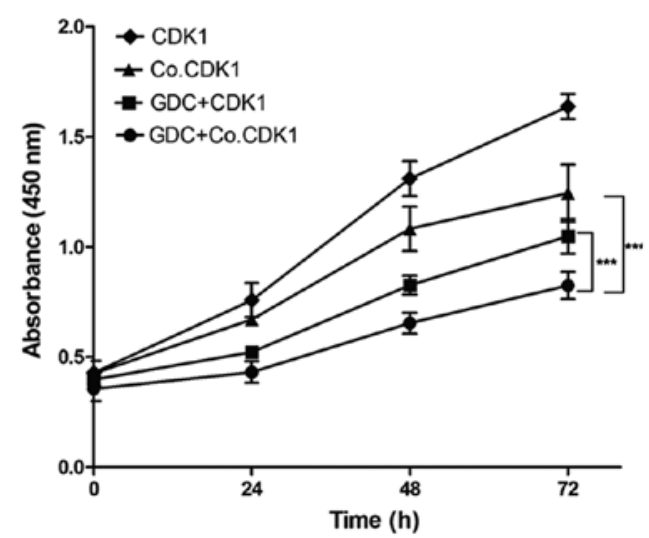

Figure 6. CDK1 was involved in the Akt signalling pathway, promoting the GBM malignancy process. (A and B) The protein level of p-Akt, cyclin A, cyclin B and CDK1 in the negative control cells and CDK1- shRNA cells, with or without the treatment of IGF-1. Protein derived from serum-starved cells treated with IGF-1 (Sigma-Aldrich; Merck KGaA) for $8 \mathrm{~h}$. ${ }^{*} \mathrm{P}<0.05,{ }^{* *} \mathrm{P}<0.01$. (C and D) The protein level of p-Akt and CDK1 in CDK1 overexpressed cells and negative control cells,treated with GDC-0491 (Selleckchem, Munich, Germany) or untreated. For up to $96 \mathrm{~h}$ treatment with $0.6 \mu \mathrm{M}$ GDC-0941, cells were collected and protein was extracted. ${ }^{*} \mathrm{P}<0.05,{ }^{* *} \mathrm{P}<0.01,{ }^{* * *} \mathrm{P}<0.0001$. (E) Treatment with GDC-0491 (GDC) markedly inhibited the proliferation of GBM cells, which was reversed by the overexpression of CDK1, ${ }^{* * *} \mathrm{P}<0.0001$. GBM, glioblastoma. $(\mathrm{F})$ The treatment of IGF-1 clearly promoted GBM cell proliferation; this was partially attenuated by the knockdown of CDK $1,{ }^{* * *} \mathrm{P}<0.0001$.

the Akt signalling pathway with IGF-1. This clearly promoted the cell proliferation of GBM, indicating that the pathway was associated with the progression of GBM. In addition, CDK1 expression was upregulated in cells treated with IGF-1 compared with in untreated cells and was downregulated in cells treated with GDC-0491 suggesting that CDK1 could be a downstream molecule of this signalling pathway in GBM. Importantly, the promotion of GBM proliferation by activation of the Akt signalling pathway was attenuated by the knockdown of CDK1 partially, and the inhibition effect of the inhibitor of this pathway was reversed by the overexpression of CDK1. These results indicated that CDK1 was involved in the Akt signalling pathway, where it promoted the GBM malignancy process. It is known that the Akt signalling pathway can promote cell cycle via inhibiting the activation of p21 (30), and that $\mathrm{p} 21$ binds to and inhibits cyclin-dependent kinase activity, preventing the phosphorylation of critical cyclin-dependent kinase substrates and blocking cell cycle progression (31). Our finding demonstrated that Akt promoted tumour cell proliferation, regulating not only the activity of the proliferation-promoting protein CDK1, 
but also its expression level. A previous study showed that the CDK1-mediated phosphorylation and stabilisation of HIF-1 $\alpha$ played a much more important role in promoting the oncogenic effects of HIF-1 in normoxic conditions than in hypoxic conditions, where CDK1 is aberrantly activated (32). Our results showed that activation of the Akt signalling pathway increased CDK1 expression. Thus, we present the Akt/CDK1/HIF-1 $\alpha$ signalling axis, and we propose that the aberrant activation of Akt is a high frequency event in GBM, which could explain the elevated HIF-1 activity observed in primary and metastatic tumours, independent of hypoxia.

In summary, using microarray datasets and bioinformatic analysis, we identified 486 DEGs, including 128 upregulated genes, that may be involved in GBM. GO function and KEGG pathway analyses showed that these upregulated DEGs may be associated with the development and progression of GBM. Upregulated genes, including CDK1,CCNB1 and CDC20, were significantly associated with the poor prognosis of patients with GBM. We focused on the relationship between the upregulated DEG CDK1 and the GBM malignancy process. The knockdown of CDK1 clearly suppressed the proliferation of GBM cells, indicating that CDK1 was involved in the Akt signalling pathway, where it promoted the GBM malignancy process. These findings improve our understanding of the fundamental mechanisms leading to GBM and may guide further research into the diagnosis and treatment of this disease.

\section{Acknowledgements}

Not applicable.

\section{Funding}

No funding was received.

\section{Availability of data and materials}

The datasets used during the present study are available from the corresponding author upon reasonable request.

\section{Authors' contributions}

YZ performed the experiments and wrote the manuscript, with the contribution of QX. JL designed the study and analysed the results. All authors read and approved the final manuscript and agreed to be accountable for all aspects of the research in ensuring that the accuracy or integrity of any part of the work are appropriately investigated and resolved.

\section{Ethics approval and consent to participate}

Not applicable.

\section{Patient consent for publication}

Not applicable.

\section{Competing interests}

The authors declare that they have no competing interests.

\section{References}

1. Dolecek TA, Propp JM, Stroup NE and Kruchko C: CBTRUS statistical report: Primary brain and central nervous system tumors diagnosed in the United States in 2005-2009. Neuro Oncol 14 (Suppl 5): v1-v49, 2012.

2. Alexander BM and Cloughesy TF: Adult glioblastoma. J Clin Oncol 35: 2402-2409, 2017.

3. Jovčevska I,Kočevar N and Komel R: Glioma and glioblastoma-how much do we (not) know? Mol Clin Oncol 1: 935-941, 2013.

4. Moreno M, Pedrosa L, Paré L, Pineda E, Bejarano L, Martínez J, Balasubramaniyan V, Ezhilarasan R, Kallarackal N, Kim SH, et al: GPR56/ADGRG1 inhibits mesenchymal differentiation and radioresistance in glioblastoma. Cell Rep 21: 2183-2197, 2017.

5. Chen Q, Cai J, Wang Q, Wang Y, Liu M, Yang J, Zhou J, Kang C, Li M and Jiang C: Long non-coding RNA NEAT1, regulated by the EGFR pathway, contributes to glioblastoma progression through the WNT/ $\beta$-Catenin pathway by scaffolding EZH2. Clin Cancer Res 24: 684-695, 2018.

6. Wang W, Zhao Z, Wu F, Wang H, Wang J, Lan Q and Zhao J: Bioinformatic analysis of gene expression and methylation regulation in glioblastoma. J Neurooncol 136: 495-503, 2017.

7. Malumbres M: Cyclin-dependent kinases. Genome Biol 15: 122, 2014.

8. Malumbres M and Barbacid M: Cell cycle, CDKs and cancer: A changing paradigm. Nat Rev Cancer 9: 153-166, 2009.

9. Xi Q, Huang M, Wang Y, Zhong J, Liu R, Xu G, Jiang L, Wang J, Fang Z and Yang S: The expression of CDK1 is associated with proliferation and can be a prognostic factor in epithelial ovarian cancer. Tumour Biol 36: 4939-4948, 2015.

10. Sung WW, Lin YM, Wu PR, Yen HH, Lai HW, Su TC, Huang RH, Wen CK, Chen CY, Chen CJ and Yeh KT: High nuclear/cytoplasmic ratio of $\mathrm{Cdk} 1$ expression predicts poor prognosis in colorectal cancer patients. BMC Cancer 14: 951, 2014.

11. Kim SJ, Nakayama S, Miyoshi Y, Taguchi T, Tamaki Y, Matsushima T, Torikoshi Y, Tanaka S, Yoshida T, Ishihara H and Noguchi S: Determination of the specific activity of CDK1 and CDK2 as a novel prognostic indicator for early breast cancer. Ann Oncol 19: 68-72, 2008.

12. Griesinger AM, Birks DK, Donson AM, Amani V, Hoffman LM, Waziri A, Wang $M$, Handler $M H$ and Foreman NK: Characterization of distinct immunophenotypes across pediatric brain tumor types. J Immunol 191: 4880-4888, 2013.

13. Wang X, Kang DD, Shen K, Song C, Lu S, Chang LC, Liao SG, Huo Z, Tang S, Ding Y, et al: An R package suite for microarray meta-analysis in quality control, differentially expressed gene analysis and pathway enrichment detection. Bioinformatics 28: 2534-2536, 2012.

14. Ritchie ME, Phipson B, Wu D, Hu Y, Law CW, Shi W and Smyth GK: Limma powers differential expression analyses for RNA-sequencing and microarray studies. Nucleic Acids Res 43: e47, 2015.

15. Gene Ontology Consortium: Gene Ontology Consortium: Going forward. Nucleic Acids Res 43: D1049-D1056, 2015.

16. Majewska E and Szeliga M: AKT/GSK3 $\beta$ signaling in glioblastoma. Neurochem Res 42: 918-924, 2017.

17. Chen Q, Li W, Wan Y, Xia X, Wu Q, Chen Y, Lai Z, Yu C and Li W: Amplified in breast cancer 1 enhances human cholangiocarcinoma growth and chemoresistance by simultaneous activation of Akt and Nrf2 pathways. Hepatology 55: 1820-1829, 2012.

18. Dolma S, Selvadurai HJ, Lan X, Lee L, Kushida M, Voisin V, Whetstone H, So M, Aviv T, Park N, et al: Inhibition of dopamine receptor D4 impedes autophagic flux, proliferation, and survival of glioblastoma stem cells. Cancer Cell 29: 859-873, 2016.

19. Rapp C, Warta R, Stamova S, Nowrouzi A, Geisenberger C, Gal Z, Roesch S, Dettling S, Juenger S, Bucur M, et al: Identification of $T$ cell target antigens in glioblastoma stem-like cells using an integrated proteomics-based approach in patient specimens. Acta Neuropathol 134: 297-316, 2017.

20. Shapiro GI: Cyclin-dependent kinase pathways as targets for cancer treatment. J Clin Oncol 24: 1770-1783, 2006.

21. Chen X, Zhang FH, Chen QE, Wang YY, Wang YL, He JC and Zhou J: The clinical significance of CDK1 expression in oral squamous cell carcinoma. Med Oral Patol Oral Cir Bucal 20: e7-e12, 2015. 
22. Zhang C, Elkahloun AG, Robertson M, Gills JJ, Tsurutani J, Shih JH, Fukuoka J, Hollander MC, Harris CC, Travis WD, et al: Loss of cytoplasmic CDK1 predicts poor survival in human lung cancer and confers chemotherapeutic resistance. PloS One 6: e23849, 2011.

23. Tsaur I, Makarević J, Hudak L, Juengel E, Kurosch M, Wiesner C, Bartsch G, Harder S, Haferkamp A and Blaheta RA: The cdk1-cyclin B complex is involved in everolimus triggered resistance in the PC3 prostate cancer cell line. Cancer Lett 313: 84-90, 2011.

24. Willder JM, Heng SJ, McCall P, Adams CE, Tannahill C, Fyffe G, Seywright M, Horgan PG, Leung HY, Underwood MA and Edwards J: Androgen receptor phosphorylation at serine 515 by Cdk1 predicts biochemical relapse in prostate cancer patients. Br J Cancer 108: 139-148, 2013.

25. Zeestraten EC, Maak M, Shibayama M, Schuster T, Nitsche U, Matsushima T, Nakayama S, Gohda K, Friess H, van de Velde CJ, et al: Specific activity of cyclin-dependent kinase $\mathrm{I}$ is a new potential predictor of tumour recurrence in stage II colon cancer. Br J Cancer 106: 133-140, 2012.

26. Hers I, Vincent EE and Tavare JM: Akt signalling in health and disease. Cell Signal 23: 1515-1527, 2011.

27. Martini M, De Santis MC, Braccini L, Gulluni F and Hirsch E PI3K/AKT signaling pathway and cancer: An updated review. Ann Med 46: 372-383, 2014
28. Guo D, Reinitz F, Youssef M, Hong C, Nathanson D, Akhavan D, Kuga D, Amzajerdi AN, Soto H, Zhu S, et al: An LXR agonist promotes glioblastoma cell death through inhibition of an EGFR/AKT/SREBP-1/LDLR-dependent pathway. Cancer Discov 1: 442-456, 2011.

29. Li X, Wu C, Chen N, Gu H, Yen A, Cao L, Wang E and Wang L: PI3K/Akt/mTOR signaling pathway and targeted therapy for glioblastoma. Oncotarget 7: 33440-33450, 2016.

30. Manning BD and Cantley LC: AKT/PKB signaling: Navigating downstream. Cell 129: 1261-1274, 2007.

31. Pestell RG, Albanese C, Reutens AT, Segall JE, Lee RJ and Arnold A: The cyclins and cyclin-dependent kinase inhibitors in hormonal regulation of proliferation and differentiation. Endocr Rev 20: 501-534, 1999.

32. Warfel NA, Dolloff NG, Dicker DT, Malysz J and El-Deiry WS: CDK 1 stabilizes HIF- $\alpha$ via direct phosphorylation of Ser668 to promote tumor growth. Cell Cycle 12: 3689-3701.

This work is licensed under a Creative Commons

Attribution-NonCommercial-NoDerivatives 4.0 International (CC BY-NC-ND 4.0) License. 